\title{
Posttransient shifts in auditory lateralization
}

\author{
YOAV ARIEH \\ Montclair State University, Montclair, New Jersey \\ AND \\ LAWRENCE E. MARKS \\ Yale University, New Haven, Connecticut
}

\begin{abstract}
Presenting an intense (e.g., 80-dB [SPL]) "transient" (e.g., 50-msec) inducer to the ear reduces the loudness of subsequent signals at or near the frequency of the inducer. In this study, we ask whether similar inducers also affect lateralization. In two experiments, we asked how inducing tones presented to one ear (the exposed ear) affect judgments of the lateral position of subsequent target tones having various interaural intensity differences. In Experiment 1, inducers had the same frequency as the targets, and, as predicted, reduced the tendency to lateralize the targets to the exposed ear. In Experiment 2, the frequency of the inducers and the target differed (different critical bands), thereby eliminating the effect on lateralization. These results are consistent with the hypothesis that inducers temporarily reduce the magnitude of the representation of intensity signals in the frequency region around them and that this reduction occurs, at least partly, peripherally to the site at which binaural intensity differences are encoded. The results imply further that the reduction in loudness previously reported under similar stimulus conditions reflects a more general reduction of intensity-based information in hearing.
\end{abstract}

It is well established that the perception of sound intensity can be altered by prior exposure to auditory stimulation. In forward masking, the threshold of a target tone increases after exposure to an inducing tone (Fastl, 1979; Plomp, 1964), and in auditory fatigue, threshold increases and loudness decreases after exposure to intense and sustained stimulation (Botte \& Mönikheim, 1994; Ward, 1973). Recent research, however, has revealed a new auditory aftereffect, one apparently mediated by a mechanism different from forward masking or auditory fatigue. Consider the following example (Arieh \& Marks, $2003 \mathrm{~b}$ ). In a baseline condition, the loudness of a $2500-\mathrm{Hz}$ target tone at $60 \mathrm{~dB}$ (SPL) matches the loudness of a 500$\mathrm{Hz}$ comparison tone at $61 \mathrm{~dB}$ (SPL). In the experimental condition, an 80-dB (SPL), 2500-Hz inducer precedes the target and reduces its loudness by $10 \mathrm{~dB}$, or $50 \%$ in sones. That is, the target now matches a $500-\mathrm{Hz}$ comparison at $51 \mathrm{~dB}$ (SPL). In contrast to auditory fatigue, the inducer is only moderately intense and, more importantly, can be as brief as $50 \mathrm{msec}$. And, in contrast to forward masking, the inducer affects only suprathreshold but not threshold-level targets (Mapes-Riordan \& Yost, 1999).

Previous research has focused exclusively on how brief inducers affect the loudness of subsequent targets. This approach is apparent in the labels that have been given to the phenomenon: first "loudness recalibration" (Marks, 1994) and later "induced loudness reduction," or ILR (Scharf, Buus, \& Nieder, 2002). Although we use ILR here, for expository purposes, the present study offers compelling evidence that the effect of the brief inducers (which for convenience we will dub "transients") on auditory perception extends beyond loudness. More specifically, we show that exposing one ear to transient inducers alters the way that listeners lateralize subsequent targets.

Motivating this study is the hypothesis that transient auditory inducers temporarily reduce the underlying sensory representation of auditory intensity in the frequency region around them and that this reduction occurs, at least partly, peripherally to the integration of neural signals from the two ears. To summarize the evidence thus far: First, from a study that measured response times, we concluded that ILR represents a decrease in the magnitude of the suprathreshold sensory responsiveness rather than a decisional shift in response criteria (Arieh \& Marks, 2003a). When listeners were asked to classify weak tones according to their frequency (high vs. low) in a speeded response task, stimulus conditions that were shown to produce ILR also produced longer response times and higher error rates. The positive relation between response time and error rate is usually interpreted as change in suprathreshold responsiveness (Wickelgren, 1977). Second, ILR transcends a particular measurement method, being observed in loudness matching (Mapes-Riordan \& Yost, 1999; Nieder, Buus, Florentine, \& Scharf, 2003), magnitude estimation (Marks, 1988, 1994; Wagner \& Scharf, 2006), judgments of loudness differences (Parker \& Schneider, 1994; Schneider \& Parker, 1990), and response time (Arieh \& Marks, 2003a). Third, ILR is fast to

Y.Arieh, ariehy@mail.montclair.edu 
set in but slow to dissipate: A significant amount of ILR was measured $225 \mathrm{msec}$ after the onset of the inducer and was still evident dozen of seconds later (Arieh, Kelly, \& Marks, 2005; Arieh \& Marks, 2003b; Wagner \& Scharf, 2006). This time course is similar to processes of peripheral sensory adaptation in other modalities - such as, in vision, processes of rapid light adaptation but slower dark adaptation (e.g., Mahroo \& Lamb, 2004).

A critical feature of ILR is the way it depends on the relative frequencies of inducer and target, which follows the predictions of the filter (critical band) model in hearing (Zwicker \& Scharf, 1965). ILR is maximal when the inducer and the target have the same frequency, $\Delta f=0$, decreasing as $\Delta f$ increases until ILR asymptotes at zero when $\Delta f$ exceeds roughly critical bandwidth (Marks, 1994; Marks \& Warner, 1991). Because frequency specificity characterizes peripheral as well as central auditory neurons (see, e.g., Schreiner \& Langner, 1988), ILR could arise centrally, peripherally, or both. Data on interaural transfer (IT) of ILR suggest both sources.

Evidence to date suggests partial transfer of ILR between the ears (Marks, 1996). In that study, subjects heard (and judged) monotic inducers, and then compared the loudness of monotic $500-\mathrm{Hz}$ and $2500-\mathrm{Hz}$ tones presented to the same (ipsilateral) and the opposite (contralateral) ear as the inducers. As measured by loudness comparison, ILR in the contralateral ear equaled $5.3 \mathrm{~dB}$, in comparison with $9.5 \mathrm{~dB}$ in the ipsilateral ear (for similar results, see also Scharf, Nieder, Buus, \& Cazals, 2003). Thus, according to a simple model that assumes independence and additivity, the magnitudes of the central and peripheral components of ILR were 5.3 and $4.2 \mathrm{~dB}$, respectively. These results imply that, although ILR partly arises from or involves neural processes central to the locus of binaural integration, a substantial component of ILR is peripheral and specific to the exposed ear.

In sum, the extant evidence supports the view that ILR (1) reflects a frequency-specific reduction in the underlying sensory representation of auditory intensity and (2) has both central and peripheral sources. If so, then it is plausible that intensity-based auditory processes other than loudness will be affected by exposure to transient inducers. In particular, the observation that ILR shows only partial IT led us to hypothesize that exposing one ear to a series of inducers will reduce the sensory representation of auditory intensity primarily at the exposed ear, thereby modifying auditory localization. Sound localization - or lateralization, when tones are presented through headphones and thus "localized" inside the head - relies not only on interaural temporal differences (phase, arrival time) but also on interaural differences in intensity: Sounds tend to be heard at the ear where SPL is greater. Thus, if an inducer produces greater ILR in the ipsilateral (exposed) ear than in the contralateral ear, inducers should also reduce the tendency to lateralize targets at the exposed ear. Experiment 1 tested this prediction.

\section{EXPERIMENT 1}

To examine the effect of transient inducers on lateralization, Experiment 1 tested subjects in three conditions. In the first condition, we measured a baseline lateralization function for each subject. We asked subjects to lateralize a series of pure tones either to the left or to the right side. The tones were presented dichotically with respect to intensity: Different dB levels were presented at the right ear and at the left ear in order to vary the target's perceived lateral position (cf. Irvine, 1987). In the second condition, we presented the same targets, but at the start of the session and every 15 trials thereafter, a series of brief, moderately loud inducers was presented to one ear. The third condition was similar to the second, but the exposed and unexposed ears were reversed. Our dependent variable was the percentage of right-side lateralization responses. We predicted that exposing one ear to transient inducers would reduce the percentage lateralization responses to that ear.

\section{Method}

Subjects. Three men and 9 women (age range 18-33 years), who reported having normal hearing, took part. The subjects in this and in the following experiment were associated with the Psychology Department at Montclair State University, and received course credit for participating.

Apparatus and Stimuli. A MATLAB program running on a Dell Pentium 4 PC controlled all aspects of stimulus presentation, data collection, and online computations. Auditory signals were generated by a Tucker-Davis System 3 Real-Time Processor at a sampling frequency of $50 \mathrm{kHz}$. The signals were then appropriately attenuated in each ear's channel (Tucker-Davis PA5 module) and delivered through calibrated TDH-49 headphones (Telephonics, Farmingdale, NY) mounted in MX41/AR cushions. Responses were entered via the computer's numeric keypad.

The inducing tones lasted $50 \mathrm{msec}\left(5-\mathrm{msec} \cos ^{2}\right.$ rise and decay) and had a frequency of $2500 \mathrm{~Hz}$ at a level of $80 \mathrm{~dB}$ (SPL). The target tones had the same duration and frequency as the inducers, but the SPLs at the right and left earphones were adjusted to vary the perceived location relative to the midline, while keeping mean SPL constant [left SPL + right SPL] $/ 2=60 \mathrm{~dB}$ (SPL) for all stimuli (there was no timing differences between the ears). For example, in one stimulus, the SPL was $61 \mathrm{~dB}$ at the right ear and $59 \mathrm{~dB}$ at the left. In this case, we expected that listeners would tend to judge the stimulus to be on the right more often than the left. Overall, we created six dichotic stimuli, three of which should be lateralized to the right side and three to the left side (right minus left ear $\mathrm{dB}$ differences of $-4,-2,-1,1,2$, and $4 \mathrm{~dB}$ ).

Procedure. The subjects were tested individually in a soundattenuated room. Each subject served in three experimental conditions: baseline, right ear induction, and left ear induction. In the baseline condition, the subjects were told that on each trial, a tone would be presented through the headphones and that their task was to decide and indicate, by pressing the appropriate key on the computer's keypad, whether the tone was perceived to the left or to the right side. After an intertrial interval of $1 \mathrm{sec}$, the next stimulus was presented. The six stimuli were presented 50 times each and the resulting 300 trials were given in a different random order to each listener. After 10 practice trials, the subjects performed in five blocks of 60 trials each.

The left ear and the right ear induction conditions were similar to the baseline condition, with one defining difference. At the start of the induction condition, and every 15 trials thereafter, 10 inducer tones were played to the left or to the right ear at a rate of $1 / \mathrm{sec}$. Presentation of the inducers was accompanied by a visual countdown on the computer screen that served to alert the listener to the imminent presentation of the subsequent (target) tone, whose lateral position was to be judged. To minimize carryover effects, the three experimental conditions were given in the course of two sessions that were separated by at least $24 \mathrm{~h}$. The baseline condition was al- 
ways given first, followed by one of the two induction conditions. The remaining induction condition was given in the second session. The baseline condition lasted about $15 \mathrm{~min}$ and the induction condition about $20 \mathrm{~min}$.

\section{Results}

The percentage of trials in which each of the six target tones was lateralized to the right was computed for each listener and each condition. Averages appear in Figure 1. The data indicate, and this will also be true in Experiment 2 , that an interaural difference of $\pm 4 \mathrm{~dB}$, the maximum tested, was not sufficient to produce $100 \%$ left or right judgments. This result is consistent with evidence that $100 \%$ lateralization judgments require a difference of 10-12 dB (Durlach \& Colburn, 1978). For example, in the baseline condition, when the SPL was $4 \mathrm{~dB}$ greater on the right, the tone was lateralized to the right ear about $75 \%$ of the time, and when the SPL was $4 \mathrm{~dB}$ greater on the left, the tone was lateralized to the left about $73 \%(100 \%-$ $27 \%$ ). Because the dB differences at the ears varied symmetrically around zero, the grand mean at baseline should be close to $50 \%$. Indeed, at baseline, the average across the six stimuli was $50.4 \%$.

When the inducers were played to the right ear, the average percentage of tones lateralized to that ear decreased in comparison with baseline. Visual inspection suggests that the reduction in lateralization to the right in this condition is evident in all six stimuli. The average of "right ear" responses across the six stimuli when the inducers were played to the right ear was $34.6 \%$, substantially below the $50.4 \%$ observed at baseline.

When the inducers were played to the left ear, the average percentage of responses lateralized to the same ear (now, the left) also decreased in comparison with baseline (or, alternately, the percentage lateralized to the right ear increased). The average of "left ear" responses across the six stimuli when the inducers were played to the left ear was $38.8 \%$, slightly above the corresponding percentage in the right ear condition but still substantially below the $50.4 \%$ observed at baseline.

To confirm the descriptive analysis, we computed the arcsine of the averaged proportions and submitted the transformed values to a repeated measures ANOVA, with inducer condition (right ear induction, baseline, left ear induction) and stimulus difference $(-4,-2,-1,1,2$ and $4 \mathrm{~dB})$ as within-subjects variables. The main effect of condition was significant $\left[F(2,22)=22.8, M S_{\mathrm{e}}=2.79\right.$, $p<.01$ ], confirming our observation that the percentage of "right ear" responses differed across conditions. Subsequent planned contrasts showed that the right ear induction and the left ear induction conditions both differed significantly from baseline $\left[F(1,11)=21.5, M S_{\mathrm{e}}=\right.$ $1.38, p<.01$ and $F(1,11)=11.6, M S_{\mathrm{e}}=2.67, p<.01$, respectively]. To round out this analysis, we note that the main effect of stimulus difference was also significant $\left[F(5,55)=22.24, M S_{\mathrm{e}}=8.1, p<.01\right]$, but the interaction between condition and stimulus was not $[F(10,110)=$ $\left.1.57, M S_{\mathrm{e}}=2.79, p>.05\right]$.

Another way to appreciate the effect on lateralization of exposing one ear to transient inducers is to compute the point of subjective equality (PSE) in each experimental condition. The PSE in a lateralization task is the difference between SPLs at the two ears producing a sound image at the midline - that is, 50\% response at each ear. To compute the PSEs, we used the linear equations to regress the average right-side responses against the corresponding $\mathrm{dB}$ differences. Then, for each condition, we computed the $\mathrm{dB}$ difference predicted to give $50 \%$ right/left response. In each condition, the variance explained by the linear equation $\left(r^{2}\right)$ was at least $92 \%$ and was statistically significant. In the baseline condition, the $\mathrm{dB}$ difference that produced the PSE was essentially zero (the signal needed to be $0.05 \mathrm{~dB}$ greater on the left). But when inducers were presented to either ear, the sound level at the ear receiving the inducers had to exceed the level at the other ear by 2.2 or $1.9 \mathrm{~dB}$ (inducers right or left, respectively).

\section{EXPERIMENT 2}

Exposing one ear to transient inducers reduced the tendency to lateralize tones to that ear. In fact, the PSE was shifted by about $2 \mathrm{~dB}$ for each ear. The results of Experiment 1 are consistent with our hypothesis that exposure to moderately loud, brief tones not only reduces loudness

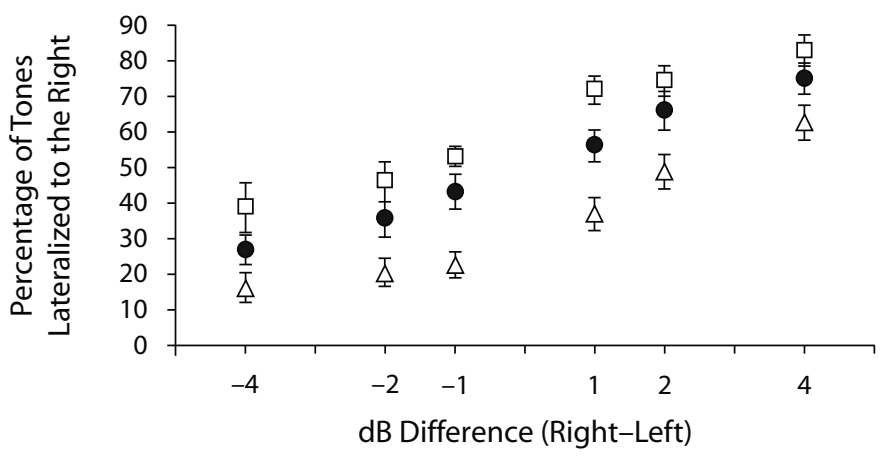

Figure 1. The percentage of right lateralization responses in Experiment 1 computed for each stimulus condition in the baseline (filled circles), right induction (triangles), and left induction (squares) conditions. Error bars indicate one standard error of the mean. 
but produces a general reduction of intensity information. Thus, the results imply the presence not only of ILR but also of induced lateralization shift (ILS). However, before we can attribute the shift in lateralization tendencies to the same mechanism that underlies ILR, we need to eliminate an alternative explanation of the results - namely, that the shift in lateralization reflects a response bias.

It is conceivable that playing a relatively loud sequence of tones to one ear biases the listeners to lateralize subsequent tones to the contralateral ear. ILR shows frequency selectivity; that is, transient inducers affect the loudness of subsequently presented targets only if the latter fall inside the inducer-critical band. Consequently, we predicted that if ILR and the shift in lateralization reported in Experiment 1 are a product of the same mechanism, than selecting the frequency of the inducer so that $\Delta f$ between inducer and target exceeds critical bandwidth should eliminate, or at least significantly reduce, the inducer's effect on lateralization. Experiment 2 tested this prediction.

\section{Method}

Subjects. Two men and 10 women (age range 18-42 years) who reported normal hearing took part. None had participated in Experiment 1.

Stimuli and Procedure. The procedure and the stimuli used in Experiment 2 were identical to those used in Experiment 1, with one important difference. In Experiment 2, the frequency of the inducer tones was $500 \mathrm{~Hz}$ instead of $2500 \mathrm{~Hz}$.

\section{Results}

The average percentage of "right ear" responses for each stimulus in each condition appears in Figure 2. In contrast to the stark differences among the lateralization functions in Experiment 1 (see Figure 1), the functions for the three conditions in Experiment 2 nearly overlap. This parity suggests that the 500-Hz inducers in Experiment 2 did not affect lateralization performance substantially. The grand means of lateralization responses to the treated ear were $49.8 \%, 48.6 \%$, and $44.1 \%$ for the baseline, right ear induction, and left ear induction conditions, respectively. The tendency to lateralize tones to the exposed ear did decrease somewhat in comparison with baseline, but the decrease is relatively small and might reflect, for some subjects, a minor bias to lateralize tones to the unexposed ear.

An ANOVA performed on the arcsine transformed values, with condition and stimulus difference as withinsubjects variables, showed that the main effect of condition was not statistically significant $\left[F(2,22)=2.7, M S_{\mathrm{e}}=0.27\right.$, $p>.05]$. Changing the frequency of the inducers tones to $500 \mathrm{~Hz}$ reduced, and almost eliminated, their effect on the lateralization of $2500-\mathrm{Hz}$ target tones. Of course, the main effect of stimulus difference was significant $[F(5,55)=$ 24.6, $\left.M S_{\mathrm{e}}=6.79, p<.01\right]$, but the interaction between condition and stimulus was not $\left[F(10,110)=2.26, M S_{\mathrm{e}}=\right.$ $0.042, p>.05]$.

The PSE was again determined by regression (the explained variances here were all at least $97 \%$, and all regressions were significant). In the baseline condition, the $\mathrm{dB}$ difference at PSE was nil $(0.01 \mathrm{~dB}$ greater on the left side), whereas in the induction conditions, the differences had to be greater at the treated right ear by $0.25 \mathrm{~dB}$ and at the treated left ear by $0.6 \mathrm{~dB}$.

To compare directly the results obtained when the frequency of the inducer was the same or different from that of the test stimuli (Experiment 1 vs. Experiment 2), we performed a global ANOVA, using frequency of inducer (same or different) as a between-subjects variable and induction condition and stimulus difference as withinsubjects variables. The results showed a significant interaction between frequency of the inducer and induction condition $\left[F(2,44)=6.9, M S_{\mathrm{e}}=0.70, p<.01\right]$, indicating that the inducer affected lateralization only when its frequency matched that of the targets.

\section{Discussion}

The aftereffects on auditory perception of presenting short, moderately loud inducers are more widespread than heretofore understood. In Experiment 1, we showed that exposing one ear to a series of inducers affects the lateralization of subsequent targets. In comparison with baseline, where inducers were withheld, the tendency to lateralize tones to the exposed ear was reduced by approximately $13 \%$ (averaged across the two ears). The results of Experiment 2

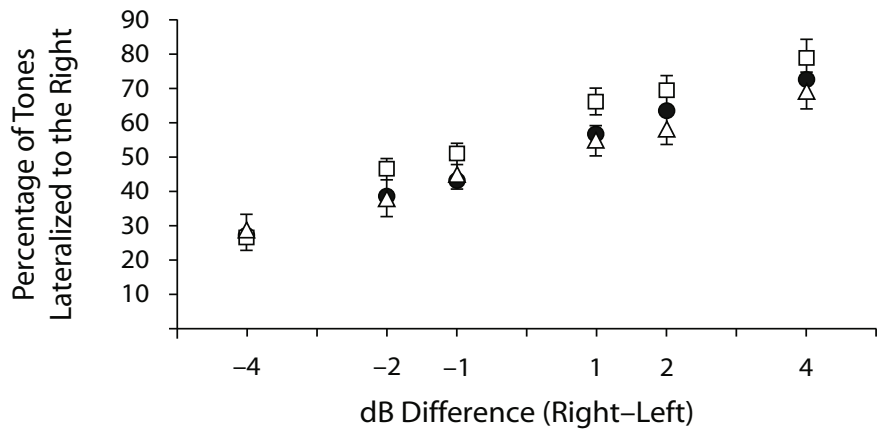

Figure 2. The percentage of right lateralization responses in Experiment 2 computed for each stimulus condition in the baseline (filled circles), right induction (triangles), and left induction (squares) conditions. Error bars indicate one standard error of the mean. 
help rule out response bias as an alternative explanation to the results. When the frequency of the inducers fell outside the critical band of the target, the effect on lateralization responses was negligible. We do recognize that those results can still be accounted for by assuming a more complex response bias that is frequency specific. Because the ILS reported here bears close affinity to ILR, and in fact, its presence or absence is predicted by the properties of ILR, we believe that this interpretation is less plausible.

One possible mechanism that has been offered to explain the reduction in the representation of intensity involves the descending efferent pathways that project from the medial olivocochlear neurons to the outer hair cells (Nieder et al., 2003). Feedback through the cochlear efferent system reduces vibration at the basilar membrane (Russell \& Murugasu, 1997) and activity in the auditory nerve (Guinan \& Stankovic, 1996) in response to stimulation. Importantly, the fact that the efferent system responds more vigorously to ipsilateral than to contralateral stimulation (Liberman, 1988) is consistent with evidence that the reduction of loudness is greater when induced ipsilaterally rather than contralaterally, and with the shift in lateralization reported here. The olivocochlear efferent system, however, produces maximum suppression at near threshold levels, where ILR is virtually absent (Mapes-Riordan \& Yost, 1999; Stankovic \& Guinan, 1999).

We offer here another possible explanation for the results in loudness and lateralization that emphasizes a general reduction of neural signals and that can be traced to peripheral and central loci. Because the shifts in lateralization produced in this study depended solely on interaural intensity relations, we suggest that the inducers reduced the magnitude of "neural intensity signals" in the exposed ear. Inducers thereby changed the effective difference between the neural signals derived from the two ears at the site where lateral position is computed, favoring the unexposed ear. Implicit in this view is the supposition that the effect of inducers on loudness, or ILR, is just one example of a much wider set of inducer-generated auditory aftereffects. If this is correct, then terms such as "induced loudness reduction" and, for that matter, "induced lateralization shift," describe but one effect of the transient inducers. A selection of a more general term, we believe, should be deferred until more is known about the underlying mechanism that mediates the effect of the inducers on loudness and lateralization.

The present results also lend support to the hypothesis that peripheral as well as central processes contribute to overall reduction in auditory intensity. Here, the terms peripheral and central are relative to the earliest site of binaural processing (superior olivary complex; Moore, 2000). In loudness, the reduction in perceived sound intensity is reported to be 9-10 dB, on average (Arieh \& Marks, 2003b; Mapes-Riordan \& Yost, 1999; Marks, 1996; Scharf et al., 2003); however, data on interaural transfer place the amount of loudness reduction at the contralateral ear at about 5-6 dB or less (Marks, 1996; Scharf et al., 2003). Those data also imply that about $4 \mathrm{~dB}$ of the magnitude of reduction is peripheral to the site where binaural information is combined. Our present results show that a series of inducers shifted the PSE by about $2 \mathrm{~dB}$. Thus, we can tentatively quantify the peripheral contribution to the shift in lateralization at $2 \mathrm{~dB}$. This value is smaller than the ear-specific component in ILR reported by Marks (1996) and Scharf et al. (2003), but this discrepancy might reflect differences in testing procedure. For example, Scharf et al. (2003) used an adaptive procedure in which the inducer tones were presented on every trial, whereas in this study, a series of inducers was presented every 15 trials. Coupled with the fact that ILR tends to accumulate over repeated presentations (Arieh et al., 2005), this difference may have led to a greater effect on loudness than on lateralization.

Loud transients have also been shown to affect identification and discrimination (Parker, Murphy, \& Schneider, 2002). In one condition, listeners were instructed to identify four weak tones (e.g., 25, 30, 35, and $40 \mathrm{~dB}$ [SPL]), and in a second condition, a fifth tone, either 90 or $45 \mathrm{~dB}$ (SPL), was added to the stimulus set. Identification accuracy decreased only when the added tone was loud. The authors attributed the reduction in accuracy to the operation of a gain-control mechanism that is under top-down control (see also Parker \& Schneider, 1994). It is not clear at this point, however, whether the effects of the inducers on loudness and on lateralization share a common mechanism with the proposed gain-control system. One discrepancy between the two observations concerns the role of listener expectancies. The reduction in identification accuracy was essentially eliminated when listeners were instructed to ignore a predictable loud tone, implying that the gain-control mechanism can be adjusted according to the experimental contingencies (Parker et al., 2002). In contrast, reduction of loudness usually occurs even when the listeners are instructed to ignore the inducer (Arieh \& Marks, 2003b).

Regardless of the mechanism or mechanisms that underlie ILR and ILS, it is notable that analogous effects are evident in modalities other than hearing: (1) in vision, in judgments of length when lines take on different orientations (Arieh \& Marks, 2002; Armstrong \& Marks, 1997) and in judgments of brightness when lights take on different colors (Marks, 1993); (2) in haptics (kinesthesis), in judgments of extent of arm movements made in different directions relative to the body (Marks \& Armstrong, 1996); (3) in taste, in judgments of intensity of structurally different compounds, such as sucrose and sodium chloride (Rankin \& Marks, 1991, 1992); and (4) in olfaction, again in judgments of intensity of structurally different compounds, such as vanillin and orange (Rankin \& Marks, 2000). Thus, the reduction in perceived intensity following exposure to transient inducers may represent a widespread characteristic of intensity processing in the nervous system. In this study, we provide evidence for induced reductions in the representation of auditory intensity that affect an auditory process other than loudness-namely, lateralization - that depends on intensity information. We will not be surprised if future research expands further the domain of induced reductions in representations of auditory intensity to still other intensity-dependent processes, such as distance estimation and speech perception. 


\section{AUTHOR NOTE}

A part of this research was presented at the 2005 Annual Meeting of the International Society for Psychophysics at Traverse City, Michigan. The authors thank Karen Kelly for help in data collection and Mark Pitt, Michael Gordon, and two anonymous reviewers for their cogent comments on an earlier version of this article. Correspondence concerning this article should be addressed to Y. Arieh, Montclair State University, Department of Psychology, Montclair, NJ 07043 (e-mail: ariehy@mail .montclair.edu).

\section{REFERENCES}

Arieh, Y., Kelly, K., \& Marks, L. E. (2005). Tracking the time to recovery after induced loudness reduction. Journal of the Acoustical Society of America, 117, 3381-3384.

ARIEH, Y., \& Marks, L. E. (2002). Context effects in visual length perception: Role of ocular, retinal, and spatial location. Perception \& Psychophysics, 64, 478-492.

ARIEH, Y., \& MARKS, L. E. (2003a). Recalibrating the auditory system: A speed-accuracy analysis of intensity perception. Journal of Experimental Psychology: Human Perception \& Performance, 29, 523-536.

ARIEH, Y., \& MarKs, L. E. (2003b). Time course of loudness recalibration: Implications for loudness enhancement. Journal of the Acoustical Society of America, 114, 1550-1556.

Armstrong, L., \& Marks, L. E. (1997). Differential effects of stimulus context on perceived length: Implications for the horizontal-vertical illusion. Perception \& Psychophysics, 59, 1200-1213.

Botтe, M. C., \& Möniкheim, S. (1994). New data on the short-term effects of tone exposure. Journal of the Acoustical Society of America, 95, 2598-2605.

Durlach, N. I., \& Colburn, H. S. (1978). Binaural phenomena. In E. C. Carterette \& M. P. Friedman (Eds.), Handbook of perception: Vol. 4., Hearing (pp. 365-466). New York: Academic Press.

FASTL, H. (1979). Temporal masking effects: III. Pure tone masker. Acustica, 43, 282-294.

Guinan, J. J., \& Stankovic, K. M. (1996). Medial efferent inhibition produces the largest equivalent attenuations at moderate to high sound levels in cat auditory-nerve fibers. Journal of the Acoustical Society of America, 100, 1680-1690.

Irvine, D. R. F. (1987). A comparison of two methods for the measurement of neural sensitivity to interaural intensity differences. Hearing Research, 30, 169-180.

LiBERMAN, M. C. (1988). Response properties of cochlear efferent neurons: Monaural vs. binaural stimulation and the effects of noise. Journal of Neurophysiology, 60, 1779-1798.

Mahroo, O. A., \& LAMB, T. D. (2004). Recovery of the human photopic electroretinogram after bleaching exposures: Estimation of pigment regeneration kinetics. Journal of Physiology, 15, 417-437.

Mapes-Riondan, D., \& Yost, W. A. (1999). Loudness recalibration as a function of level. Journal of the Acoustical Society of America, 106, 3506-3511.

Marks, L. E. (1988). Magnitude estimation and sensory matching. Perception \& Psychophysics, 43, 511-525.

MarKs, L. E. (1993). Differential context effects within but not between modalities? In A. Garriga-Trillo et al. (Eds.), Fechner Day 1993: Proceedings of the Ninth Annual Meeting of the International Society for Psychophysics (pp. 161-166). Madrid: UNED.

MARKs, L. E. (1994). "Recalibrating" the auditory system: The perception of loudness. Journal of Experimental Psychology: Human Perception \& Performance, 20, 382-396.

MARKS, L. E. (1996). Recalibrating the perception of loudness: Interaural transfer. Journal of the Acoustical Society of America, 100, 473-480.
Marks, L. E., \& Armstrong, L. (1996). Haptic and visual representations of space. In T. Inui \& J. L. McClelland (Eds.), Attention and performance XVI: Information integration in perception and communication (pp. 263-287). Cambridge, MA: MIT Press, Bradford Books.

MARKs, L. E., \& WARNER, E. (1991). Slippery context effect and critical bands. Journal of Experimental Psychology: Human Perception \& Performance, 17, 986-996.

Moore, J. K. (2000). Organization of the human superior olivary complex. Microscopy Research \& Technique, 15, 403-412.

Nieder, B., BuUs, S., Florentine, M., \& Scharf, B. (2003). Interactions between test- and inducer-tone durations in induced loudness reduction. Journal of the Acoustical Society of America, 114, 2846-2855.

Parker, S., Murphy, D. R., \& Schneider, B. A. (2002). Top-down gain control in the auditory system: Evidence from identification and discrimination experiments. Perception \& Psychophysics, 64, 598-615.

Parker, S., \& Schneider, B. [A.] (1994). The stimulus range effect: Evidence for top-down control of sensory intensity in audition. Perception \& Psychophysics, 56, 1-11.

Plomp, R. (1964). Rate of decay of auditory sensation. Journal of the Acoustical Society of America, 36, 277-282.

Rankin, K. M., \& MarKs, L. E. (1991). Differential context effects in taste perception. Chemical Senses, 16, 617-629.

RANKIN, K. M., \& MARKs, L. E. (1992). Effects of context on sweet and bitter tastes: Unrelated to sensitivity to PROP (6- $n$-propylthiouracil). Perception \& Psychophysics, 52, 479-486.

Rankin, K. M., \& Marks, L. E. (2000). Chemosensory context effects: Role of perceived similarity and neural commonality. Chemical Senses, 25, 747-759.

Russell, I. J., \& Murugasu, E. (1997). Medial efferent inhibition suppresses basilar membrane responses to near characteristic frequency tones of moderate to high intensities. Journal of the Acoustical Society of America, 102, 1734-1738.

Scharf, B., BuUs, S., \& Nieder, B. (2002). Loudness enhancement: Induced loudness reduction in disguise? Journal of the Acoustical Society of America, 112, 807-810.

Scharf, B., Nieder, B., Buus, S., \& Cazals, Y. (2003). Induced loudness reduction: Correlations between ears and procedures. In B. Berglund \& E. Borg (Eds.), Fechner Day 2003 (pp. 281-286). Larnaca, Cyprus: International Society for Psychophysics.

SchneIder, B. [A.], \& PARKeR, S. (1990). Does stimulus context affect loudness or only loudness judgments? Perception \& Psychophysics, 48, 409-418.

Schreiner, C. E., \& Langner, G. (1988). Periodicity coding in the inferior colliculus of the cat. II. Topographical organization. Journal of Neurophysiology, 60, 1823-1840.

Stankovic, K. M., \& Guinan, J. J. (1999). Medial efferent effects on auditory-nerve responses to tail-frequency tones: I. Rate reduction. Journal of the Acoustical Society of America, 106, 857-869.

WAGNER, E., \& SCHARF, B. (2006). Induced loudness reduction as a function of exposure time and signal frequency. Journal of the Acoustical Society of America, 119, 1012-1020.

WARD, W. D. (1973). Adaptation and fatigue. In J. Jerger (Ed.), Modern developments in audiology (Rev. ed., pp. 301-344). New York: Academic Press.

WiCKELGREN, W. A. (1977). Speed-accuracy tradeoff and information processing dynamics. Acta Psychologica, 41, 67-85.

ZWICKER, E., \& SCHARF, B. (1965). A model of loudness summation. Psychological Review, 72, 3-26.

(Manuscript received March 20, 2006; revision accepted for publication August 24, 2006.) 